\title{
in quasi-two-dimensional tri-layer $\mathrm{La}_{2.1} \mathrm{Sr}_{1.9} \mathrm{Mn}_{3} \mathrm{O}_{10}$ manganite
}

\author{
Jeetendra Kumar Tiwari, Birendra Kumar, Harish Chandr Chauhan \& Subhasis Ghosh ${ }^{\bowtie}$
}

The tri-layer $\mathrm{La}_{3-3 x} \mathrm{Sr}_{1+3 x} \mathrm{Mn}_{3} \mathrm{O}_{10}$ manganites of Ruddlesden-Popper (RP) series are naturally arranged layered structure with alternate stacking of $\omega-\mathrm{MnO}_{2}(\omega=3)$ planes and rock-salt type block layers $(\mathrm{La}, \mathrm{Sr})_{2} \mathrm{O}_{2}$ along c-axis. The dimensionality of the RP series manganites depends on the number of perovskite layers and significantly affects the magnetic and transport properties of the system. Generally, when a ferromagnetic material undergoes a magnetic phase transition from ferromagnetic to paramagnetic state, the magnetic moment of the system becomes zero above the transition temperature $\left(T_{C}\right)$. However, the tri-layer $\mathrm{La}_{2.1} \mathrm{Sr}_{1.9} \mathrm{Mn}_{3} \mathrm{O}_{10}$ shows non-zero magnetic moment above $\mathrm{T}_{C}$ and also another transition at higher temperature $T^{*} \approx 263 \mathrm{~K}$. The non-zero magnetization above $\mathrm{T}_{C}$ emphasizes that the phase transition in tri-layer $\mathrm{La}_{2.1} \mathrm{Sr}_{1.9} \mathrm{Mn}_{3} \mathrm{O}_{10}$ not a ferromagnetic to paramagnetic state. We show here the non-zero magnetic moment above $\mathrm{T}_{C}$ is due to the quasi-two-dimensional nature of the tri-layer $\mathrm{La}_{2.1} \mathrm{Sr}_{1.9} \mathrm{Mn}_{3} \mathrm{O}_{10}$ manganite. The scaling of the magnetic entropy change confirms the second-order phase transition and the critical behavior of phase transition has been studied around $\mathrm{T}_{C}$ to understand the low dimensional magnetism in tri-layer $\mathrm{La}_{2.1} \mathrm{Sr}_{1.9} \mathrm{Mn}_{3} \mathrm{O}_{10}$. We have obtained the critical exponents for tri-layer $\mathrm{La}_{2.1} \mathrm{Sr}_{1.9} \mathrm{Mn}_{3} \mathrm{O}_{10}$, which belong to the short-range two-dimensional (2D)-Ising universality class. The low dimensional magnetism in tri-layer $\mathrm{La}_{2.1} \mathrm{Sr}_{1.9}$ $\mathrm{Mn}_{3} \mathrm{O}_{10}$ manganite is also explained with the help of renormalization group theoretical approach for short-range 2D-Ising systems. It has been shown that the layered structure of tri-layer $\mathrm{La}_{2.1} \mathrm{Sr}_{1.9} \mathrm{Mn}_{3} \mathrm{O}_{10}$ results in three different types of interactions intra-planer $\left(J_{a b}\right)$, intra-tri-layer $\left(J_{c}\right)$ and inter-tri-layer $\left(J^{\prime}\right)$ such that $J_{a b}>J_{c}>>J^{\prime}$ and competition among these give rise to the canted antiferromagnetic spin structure above $T_{C}$. Based on the similar magnetic interaction in bi-layer manganite, we propose that the tri-layer $\mathrm{La}_{2.1} \mathrm{Sr}_{1.9} \mathrm{Mn}_{3} \mathrm{O}_{10}$ should be able to host the skyrmion below $\mathrm{T}_{C}$ due to its strong anisotropy and layered structure.

Physical properties of naturally layered manganites. The tri-layer $\mathrm{La}_{3-3 x} \mathrm{Sr}_{1+3 x} \mathrm{Mn}_{3} \mathrm{O}_{10}$ manganites are a member of the Ruddlesden-Popper (RP) series ( $\mathrm{La}, \mathrm{Sr})_{\omega+1} \mathrm{Mn}_{\omega} \mathrm{O}_{3 \omega+1}$ manganite perovskites, where $\omega=1$, $2,3 \ldots \infty^{1}$. The RP series manganites are naturally arranged layered structures with alternate stacking of $\omega-\mathrm{MnO}_{2}$ planes and rock-salt type block layers $(\mathrm{La}, \mathrm{Sr})_{2} \mathrm{O}_{2}$ along c-axis ${ }^{1}$. In the $\mathrm{RP}$ series manganites, the dimensionality depends on the number of perovskite layers and significantly affects the magnetic and transport properties of the system. In manganites, the introduction of a divalent atom in place of a trivalent atom causes the coexistence of $\mathrm{Mn}^{3+}$ and $\mathrm{Mn}^{4+}$ ion, which alters the bond length of $\mathrm{Mn}-\mathrm{O}$ due to the Jahn-Teller (JT) effect ${ }^{2-5}$. The $3 \mathrm{~d}$ orbital of Mn splits into two energy levels $\mathrm{t}_{2 g}$ and $\mathrm{e}_{g}$ in the presence of crystal field and JT effect. The doping of divalent atom results in some empty $e_{g}$-orbital energy levels, which facilitate hopping of electrons responsible for transport properties of tri-layer $\mathrm{La}_{3}-3 x \mathrm{Sr}_{1+3 x} \mathrm{Mn}_{3} \mathrm{O}_{10}$. The ferromagnetism (FM) and metal to insulator transition in manganites are governed by the hoping of $\mathrm{e}_{g}$-orbital electrons between the adjoining $\mathrm{Mn}^{3+}$ and $\mathrm{Mn}^{4+}$ ions through $\mathrm{O}$. This mechanism is called double exchange (DE) interaction ${ }^{6}$. The most studied oxides of the RP series manganites are bi-layer $(\omega=2) \mathrm{La}_{2-2 x} \mathrm{Sr}_{1+2 x} \mathrm{Mn}_{2} \mathrm{O}_{7}$ and infinite-layer $(\omega=\infty) \mathrm{La}_{1-x} \mathrm{Sr}_{x} \mathrm{MnO}_{3}$. In particular, the three-dimensional (3D) infinite-layer $\mathrm{La}_{1-x} \mathrm{Sr}_{x} \mathrm{MnO}_{3}$ is the most widely studied manganite perovskite due to its extraordinary thermal, electronic and magnetic properties ${ }^{2,7-16}$. The $3 \mathrm{D} \mathrm{La}_{1-x} \mathrm{Sr}_{x} \mathrm{MnO}_{3}$ manganite perovskites have continuous stacking of perovskite structure. The bi-layer $\mathrm{La}_{2}-2 x \mathrm{Sr}_{1+2 x} \mathrm{Mn}_{2} \mathrm{O}_{7}$ manganites consist of quasi-two-dimensional (Q2D) $\mathrm{MnO}_{2}$ bi-layers separated by an insulating $(\mathrm{La}, \mathrm{Sr})_{2} \mathrm{O}_{2}$ layer ${ }^{17}$ and received growing interest due to their intriguing physical properties ${ }^{13,14,17-25}$. Apart from the extraordinary magnetic and 

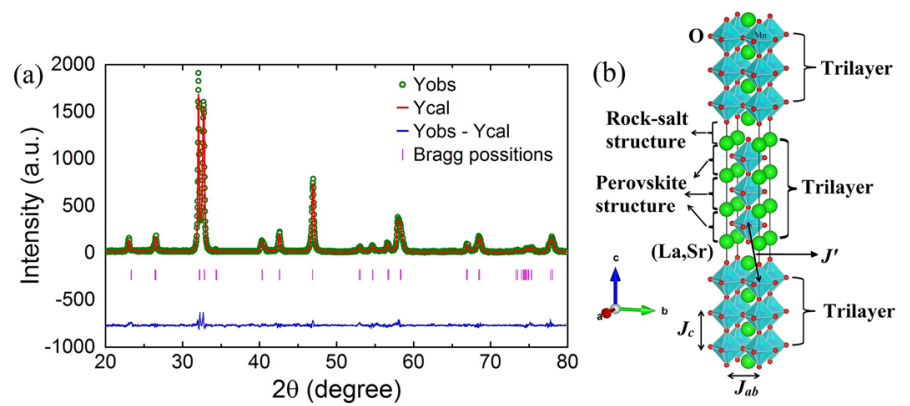

Figure 1. (a) Rietveld refinement of the single phase TL-LSMO-0.3 (OriginPro 2015 software) and (b) Unit cell crystal structure of the tri-layer TL-LSMO-0.3 (VESTA software https://jp-minerals.org/vesta/en/). The $\mathrm{MnO}_{6}$ octahedra in the crystalline bulk are denoted in cyan color. Different atoms are shown as the spheres of different [(La, Sr) - light green, $\mathrm{Mn}$-blue and oxygen-red]. The perovskite structure $\mathrm{ABO}_{3},[\mathrm{~A} \rightarrow(\mathrm{La}, \mathrm{Sr})$ and $\mathrm{B} \rightarrow$

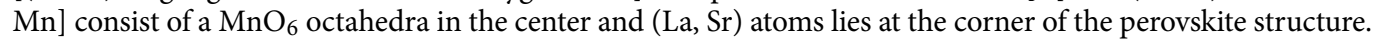

transport properties, recently observed skyrmionic-bubbles in manganite perovskites ${ }^{26-29}$ triggered the renewed attention of researchers. A magnetic skyrmion is a topological particle having a local whirl of the spins ${ }^{30-33}$. A topological skyrmion formation occurs due to the competition among different interactions such as Heisenberg (HI) interaction, Dzyaloshinskii-Moriya (DM) interaction, long-range dipole interaction and anisotropy ${ }^{26-33}$. In non-centrosymmetric magnetic materials, DM and HI interaction are responsible for skyrmion formation ${ }^{30}$. On the other hand, in centrosymmetric magnetic materials, long-range dipole interaction and anisotropy have been proposed to be responsible for the formation of the skyrmions ${ }^{26}$. Though, it is not yet fully understood how the absence of DM interaction can give rise to skyrmions in manganites. The tri-layer $\mathrm{La}_{3}-3{ }_{x} \mathrm{Sr}_{1+3 x} \mathrm{Mn}_{3} \mathrm{O}_{10}$ manganites have Q2D $\mathrm{MnO}_{2}$ tri-layers separated by $(\mathrm{La}, \mathrm{Sr})_{2} \mathrm{O}_{2}$ layer as shown in Fig. 1b. However, there are remarkably few studies on the transport andmagnetic properties of tri-layer $\mathrm{La}_{3}-3 x \mathrm{Sr}_{1+3 x} \mathrm{Mn}_{3} \mathrm{O}_{10}$ manganites. There are only two reports on tri-layer $\mathrm{La}_{3}{ }_{3 x} \mathrm{Sr}_{1+3 x} \mathrm{Mn}_{3} \mathrm{O}_{10}$ manganites ${ }^{34,35}$. Both the reports contain a very limited discussion about the structural, magnetic and transport properties of $\mathrm{La}_{2.1} \mathrm{Sr}_{1.9} \mathrm{Mn}_{3} \mathrm{O}_{10}$. The scarcity of the studies in tri-layer $\mathrm{La}_{3}-3 x \mathrm{Sr}_{1}+3 x \mathrm{Mn}_{3} \mathrm{O}_{10}$ manganites is because of the inherent difficulty in synthesizing the high-quality samples of the tri-layer manganites. The preparation of tri-layer manganite samples is challenging compared to the bi-layer $\mathrm{La}_{2-2 x} \mathrm{Sr}_{1+2 x} \mathrm{Mn}_{2} \mathrm{O}_{7}$ and infinite-layer $\mathrm{La}_{1-x} \mathrm{Sr}_{x} \mathrm{MnO}_{3}$ manganites due to difficulty in achieving the stable phase. A little mismatch in the stoichiometric ratio of the precursors and a little deviation from the required temperature cycle may result in the formation of 3D infinite-layer or Q2D bi-layer manganites perovskite as an impurity in the matrix of tri-layer manganite. Hence, careful synthesis of tri-layer $\mathrm{La}_{3}-3 x$ $\mathrm{Sr}_{1+3 x} \mathrm{Mn}_{3} \mathrm{O}_{10}$ manganite is required to get a high-quality sample without impurity. As it stands presently, the study of tri-layer $\mathrm{La}_{3}-3 x \mathrm{Sr}_{1+3 x} \mathrm{Mn}_{3} \mathrm{O}_{10}$ manganite is important due to the following issues: (i) the magnetic and transport properties are not at all explored rigorously, (ii) the exchange mechanism responsible for the spin-spin interaction for FM is not known and (iii) recently observed skyrmionic-bubbles in manganites ${ }^{26-29}$ indicates that the tri-layer $\mathrm{La}_{3}-3 x \mathrm{Sr}_{1+3 x} \mathrm{Mn}_{3} \mathrm{O}_{10}$ may also be a potential candidate for the skyrmion host material. These issues emphasize that a thorough magnetic analysis of the tri-layer $\mathrm{La}_{3}-3 x \mathrm{Sr}_{1+3 x} \mathrm{Mn}_{3} \mathrm{O}_{10}$ is required to establish the basic understanding of the magnetism and the exchange interaction involved in the tri-layer manganite. The magnetic and electrical properties are explored with the help of high precision magnetic and electrical measurements. In order to investigate the exchange mechanism responsible for the spin-spin interaction, a detailed critical analysis of the second-order phase transition has been carried out.

In the present study, we have chosen a tri-layer compound $\mathrm{La}_{2.1} \mathrm{Sr}_{1.9} \mathrm{Mn}_{3} \mathrm{O}_{10}$ for $\mathrm{x}=0.3$ (hereafter referred to as TL-LSMO-0.3, where TL-stands for tri-layer). The low dimensional magnetism in tri-layer TL-LSMO-0.3 is explained by the critical analysis using different methods, which includes Kouvel-Fisher (KF) method, modified Arrott plots (MAPs), scaling and critical isotherm analysis. Further confirmation of the low dimensionality of the magnetism in TL-LSMO-0.3 is obtained by renormalization group theory. We have shown that the layered manganite TL-LSMO-0.3 has special characteristics that cannot be explained by the $3 \mathrm{D}$ universality classes.

\section{Experimental details}

A high-quality tri-layer $\mathrm{La}_{2.1} \mathrm{Sr}_{1.9} \mathrm{Mn}_{3} \mathrm{O}_{10}$ manganite sample was synthesized through the standard solid state reaction technique. The stoichiometric amount of high purity precursors of $\mathrm{La}_{2} \mathrm{O}_{3}, \mathrm{SrCO}_{3}$ and $\mathrm{MnO}_{2}$ were grounded together to achieve the homogeneous mixture of the sample. The final mixture was then calcined at $1050{ }^{\circ} \mathrm{C}$ for $48 \mathrm{~h}$ and sintered at $1400^{\circ} \mathrm{C}$ for $72 \mathrm{~h}$ after making pallets. The sample was regrounded after each calcination and sintering, the final sintering process was repeated to achieve the single phase. The room temperature crystal structure and phase purity were determined by the powder X-ray diffraction (PXRD) (Rigaku miniflex 600-X-ray diffractometer with $\mathrm{Cu}-\mathrm{K}_{\alpha}$ radiation) followed by the Rietveld refinement. The sample was found to be a tetragonal $(I 4 / \mathrm{mmm})$ structure with no impurity peak. The temperature and field-dependent high precision magnetic data were collected using a physical property measurement system (PPMS). The temperaturedependent zero-field cooled (ZFC) and field cooled (FC) magnetization data were obtained under a constant magnetic field of $10 \mathrm{mT}$ in the temperature range 5-300 K. First quadrant field-dependent $\mathrm{M}-\mathrm{H}$ curves were obtained under a varying magnetic field of $0-7 \mathrm{~T}$ (field step is; 0 to $500 \mathrm{mT} \rightarrow \Delta \mu_{0} \mathrm{H}=20 \mathrm{mT}$ and $500 \mathrm{mT}$ to 7 


\begin{tabular}{|l|l|}
\hline Parameters & $\mathbf{L a}_{2.1} \mathbf{S r}_{1.9} \mathbf{M n}_{\mathbf{3}} \mathbf{O}_{\mathbf{1 0}}$ \\
\hline Symmetry & Tetragonal \\
\hline Space group & $I 4 / m m m$ \\
\hline $\mathrm{a}=\mathrm{b}(\AA)$ & 3.90330 \\
\hline $\mathrm{c}(\AA)$ & 28.13800 \\
\hline $\mathrm{V}\left(\AA^{3}\right)$ & 428.7035 \\
\hline $\mathrm{R}_{p}(\%)$ & 15.2 \\
\hline $\mathrm{R}_{w p}(\%)$ & 18.8 \\
\hline $\mathrm{R}_{F}(\%)$ & 5.12 \\
\hline$\chi^{2}(\%)$ & 1.98 \\
\hline
\end{tabular}

Table 1. The Rietveld refinement parameters of TL-LSMO-0.3.

$\mathrm{T} \rightarrow \Delta \mu_{0} \mathrm{H}=200 \mathrm{mT}$ ) in the temperature range of 90 to $120 \mathrm{~K}$ with $\Delta \mathrm{T}=1 \mathrm{~K}$. The resistivity of TL-LSMO-0.3 was collected in the temperature range of 10 to $300 \mathrm{~K}$ by using PPMS.Figure 1a, b show the Rietveld refinement and crystal structure of the TL-LSMO-0.3, respectively. The Rietveld refinement results of the TL-LSMO-0.3 sample are listed in Table 1. The crystal structure of TL-LSMO-0.3 shows a layered characteristic in which the rock-salt type structure separates three consecutive perovskite layers and these perovskites layers are made of a two-dimensional (2D) network of $\mathrm{Mn}-\mathrm{O}$ bond and form Q2D $\mathrm{MnO}_{2}$ planes ${ }^{23}$. In contrast, the crystal structure of infinite-layer manganites has continuous stacking of the 3D perovskite layers, which is made of a 3D network of $\mathrm{Mn}-\mathrm{O}$ bond and noticeably different than that of TL-LSMO- $0.3^{23}$. Hence, the dimensionality of the RP series manganites can be modified by altering the number of perovskite layers ${ }^{34}$.

\section{Results and analysis}

The FC and resistivity curves for TL-LSMO-0.3 are shown in Fig. $2 \mathrm{a}$. The transition temperature $\left(\mathrm{T}_{C}\right) \approx 103 \mathrm{~K}$ of the sample is determined by the minimum of the derivative of FC curve, shown in the inset (1) of Fig. $2 \mathrm{a}^{34,35}$. There is another transition $\mathrm{T}^{*}$ that appears at $\approx 263 \mathrm{~K}$ in addition to the first transition $103 \mathrm{~K}$. Generally, in the case of infinite-layer manganites, the magnetization above $\mathrm{T}_{C}$ is zero, but in the present sample, the magnetization is non-zero above $\mathrm{T}_{C}$. Similar results have been observed in bi-layer $\mathrm{La}_{2}-2 x \mathrm{Sr}_{1+2 x} \mathrm{Mn}_{2} \mathrm{O}_{7}$ manganites ${ }^{17,36,37}$. The non-zero magnetization above $\mathrm{T}_{C}$ in bi-layer $\mathrm{La}_{2-2 x} \mathrm{Sr}_{1+2 x} \mathrm{Mn}_{2} \mathrm{O}_{7}$ manganites has been explained by the 2D short-range FM ordering in their PM state ${ }^{17,36,37}$. The temperature-dependent resistivity curve for TL-LSMO-0.3 shows a broad peak at $\approx 230 \mathrm{~K}$ corresponding to the metal-insulator transition $\left(\mathrm{T}_{M I}\right)$ along with a step-like behavior at $\mathrm{T}_{C} \approx 103 \mathrm{~K}$ [inset (2)]. At present, we have not any clear explanation of this huge difference in $\mathrm{T}_{C}$ and $\mathrm{T}_{M I}$, it is a course of future study. Figure $2 \mathrm{~b}$ represents the $\mathrm{M}-\mathrm{H}$ data of TL-LSMO-0.3 in critical region 90 $\mathrm{K} \leq \mathrm{T} \leq 120 \mathrm{~K}$, where $\Delta \mathrm{T}=1 \mathrm{~K}$.

\section{Entropy analysis}

Universal curve for second-order phase transition. This section presents a systematic study of the behavior of the universal curve for magnetic entropy change $\left(\Delta S_{M}\right)$ to confirm the order of magnetic phase transition in TL-LSMO-0.3. A universal curve should be constructed for field-dependent $\Delta S_{M}$ only in case of second-order phase transition ${ }^{38-42}$. The existence of a universal curve is based on the formalism that the equivalent points of the different $\Delta S_{M}$ curves calculated for different magnetic fields should collapse on a single curve $^{38-42}$. If TL-LSMO-0.3 shows a second-order magnetic phase transition, all the $\Delta S_{M}$ curves will collapse on a single universal curve. Before starting the analysis of the scaling behavior of $\Delta S_{M}$, we have to calculate the temperature variation of $\Delta S_{M}$. The $\Delta S_{M}$ can be calculated by using $\mathrm{M}-\mathrm{H}$ data and Maxwell's thermodynamic relation given below ${ }^{39,43}$

$$
\begin{aligned}
& \Delta S_{M}\left(\frac{T_{1}+T_{2}}{2}\right)=\frac{1}{\left(T_{1}-T_{2}\right)}\left[\int _ { 0 } ^ { \mu _ { 0 } H _ { \operatorname { m a x } } } \left(\partial M\left(\mu_{0} H, T_{2}\right)\right.\right. \\
& \left.-\partial M\left(\mu_{0} H, T_{1}\right)_{\mu_{0} H} d\left(\mu_{0} H\right)\right]
\end{aligned}
$$

where $\mu_{0}, \mathrm{H}$ and $\mathrm{M}$ are the vacuum permeability, magnetic field and magnetization, respectively. Figure 3 a shows the variation of $\Delta S_{M}$ with temperature and all the curves show a maximum at $\mathrm{T}_{C}$. The value of the $\Delta S_{M}$ peak increases with the magnetic field. In order to construct the universal curve, all the $\Delta S_{M}$ curves were normalized with their respective maximum entropy change $\Delta S_{M}\left(T, \mu_{0} H\right) / \Delta S_{M}^{p e a k}\left(\mathrm{~T}, \mu_{0} \mathrm{H}\right)$. Next, the temperature axis is rescaled by considering the reference temperature such that $\Delta S_{M}\left(T_{r}\right) / \Delta S_{M}^{p e a k} \geq l$, where $\mathrm{T}_{r}$ is the reference temperature and $l(0<l \leq 1)$ is the arbitrary constant. Although $l$ can take any value between 0 to 1 , the but large value of $l$, i.e., the reference temperature chosen very close to $\Delta S_{M}^{\text {peak }}$ may result in a large numerical error due to the limited number of points. We define the new rescaled temperature axis $(\theta)$ as

$$
\theta=\left\{\begin{array}{l}
-\left(T-T_{C}\right) /\left(T_{r_{1}}-T_{C}\right), T \leq T_{C} \\
\left(T-T_{C}\right) /\left(T_{r_{2}}-T_{C}\right), T>T_{C},
\end{array}\right.
$$



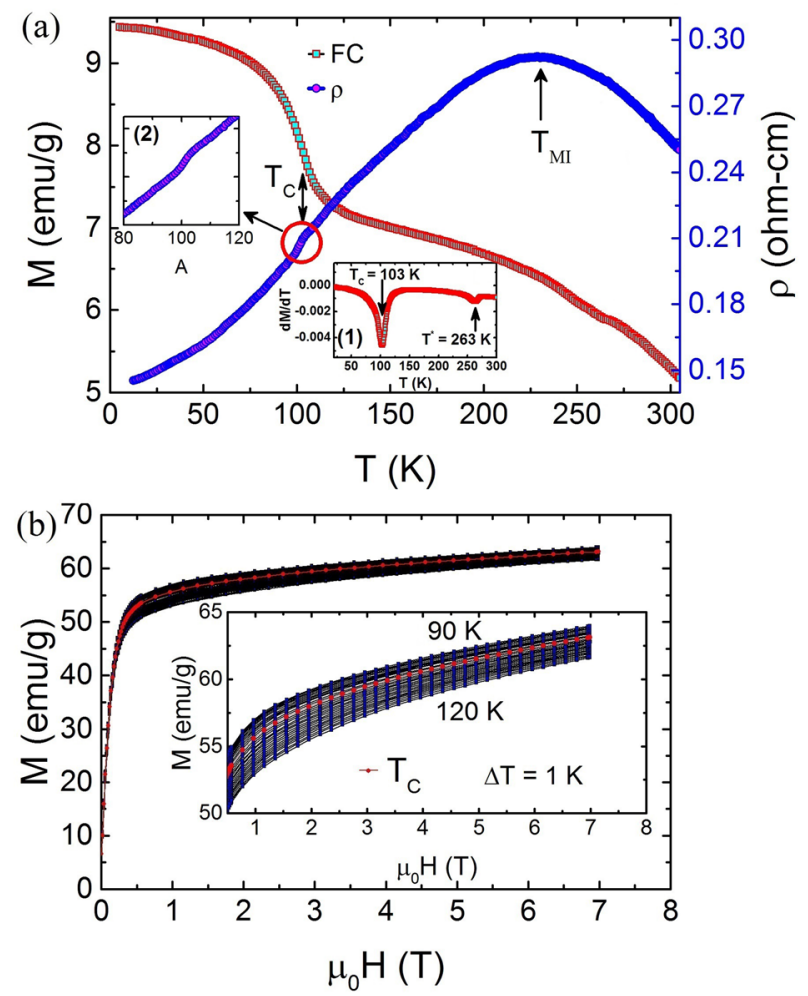

Figure 2. (a) FC of TL-LSMO-0.3 in constant magnetic field of $10 \mathrm{mT}$. The $\mathrm{T}_{C} \approx 103 \mathrm{~K}$ is determined by the minimum of the derivative of magnetization $(\mathrm{dM} / \mathrm{dT})$. Resistivity of TL-LSMO-0.3 shows a metal-insulator transition $\left(\mathrm{T}_{M I}\right)$ at $\approx 230 \mathrm{~K}$ and a step at $\approx 103 \mathrm{~K}$ corresponding to the magnetic phase transition $\mathrm{T}_{C}$ and a anomaly at $\approx 263 \mathrm{~K}$ represented by $\mathrm{T}^{*}$. (b) M-H curve for TL-LSMO- 0.3 in the temperature range 90 to $120 \mathrm{~K}$ with $\Delta \mathrm{T}=1 \mathrm{~K}$ and magnetic field of $7 \mathrm{~T}$. (Figures have been drawn using OriginPro 2015 software).

where $\mathrm{T}_{r_{1}}$ and $\mathrm{T}_{r_{2}}$ are the two reference temperatures for $\mathrm{T} \leq \mathrm{T}_{C}$ and $\mathrm{T}>\mathrm{T}_{C}$, respectively. The reference temperatures $\mathrm{T} \leq \mathrm{T}_{C}$ and $\mathrm{T}>\mathrm{T}_{C}$ are selected such that $\Delta S_{M}\left(T_{r_{1}}\right) / \Delta S_{M}^{\text {peak }}=\Delta S_{M}\left(T_{r_{2}}\right) / \Delta S_{M}^{\text {peak }}=0.7$. The universal curve for TL-LSMO-0.3 is plotted in Fig. 3b, which shows the collapse of all the $\Delta S_{M}$ curves calculated at the different fields on a single curve. The formation of a single curve for TL-LSMO-0.3 confirms the second-order phase transition around $\mathrm{T}_{C}$.

\section{Critical exponent analysis}

One way to determine the $\mathrm{T}_{C}$ and critical exponents $(\beta$ and $\gamma)$ is the Arrott analysis of the data ${ }^{44}$. Generally, if a magnetic system belongs to the mean-field ordering, i.e., $\beta=0.5, \gamma=1$, then Arrott plot $\left(\mathrm{M}^{2}\right.$ vs. $\left.\mu_{0} \mathrm{H} / \mathrm{M}\right)$ should result in parallel lines and the isothermal curve at $\mathrm{T}_{C}$ should pass through the origin ${ }^{44}$. The Arrot plot $\left(\mathrm{M}^{2}\right.$ vs. $\mu_{0}$ $\mathrm{H} / \mathrm{M}$ ) for TL-LSMO-0.3 does not yield parallel lines around $\mathrm{T}_{C}$, as shown in Fig. $4 \mathrm{a}$, which implies that there is non-mean-field type interaction in TL-LSMO-0.3. The positive slope in the Arrott plot confirms the second-order magnetic phase transition in TL-LSMO- $0.3^{45}$. Neither the short-range 3D-Ising model $(\beta=0.325, \gamma=1.24)$ nor the long-range $2 \mathrm{D}$-Ising model $(\beta=0.289, \gamma=1.49)$ produces parallel lines, shown in Fig. $4 \mathrm{~b}, \mathrm{c}$. Therefore one can conclude that these two models cannot describe the critical behavior of TL-LSMO-0.3. Hence, we reanalyzed the magnetization isotherms of the TL-LSMO-0.3 by using the Arrott Noakes equation of state defined in the critical region, Eq. (11) ${ }^{46}$. The modified Arrott plots (MAPs) $\mathrm{M}^{1 / \beta}$ vs. $\mu_{0} \mathrm{H} / \mathrm{M}^{1 / \gamma}$ for the $\mathrm{M}-\mathrm{H}$ isotherms of TLLSMO-0.3 in the asymptotic region $\left[\left|\epsilon=\left(T-T_{c}\right) / T_{c}\right|<0.1\right.$, where $\epsilon$ is the reduced temperature] is shown in Fig. $4 \mathrm{~d}$. The value of the exponents $\beta$ and $\gamma$ are chosen such that the isotherms of MAPs display as close as parallel lines. The best fit of Eq. (11) to the MAPs defined for TL-LSMO-0.3 in the temperature range $95 \mathrm{~K} \leq$ $\mathrm{T} \leq 111 \mathrm{~K}$ and field range $0.5 \mathrm{~T} \leq \mu_{0} \mathrm{H} \leq 7 \mathrm{~T}$ yields the value of exponents $\beta=0.118 \pm 0.004, \gamma=1.681 \pm 0.006$ and $\mathrm{T}_{C}=103.54 \pm 0.03 \mathrm{~K}$.

Next, we find out the value of exponent $\delta$ using M-H curve at $\mathrm{T}_{C}$ and Eq. (10) as shown in Fig. 5. The value of the exponent $\delta=14.668 \pm 0.002$ is obtained for TL-LSMO- 0.3 by fitting the isotherm at $\mathrm{T}_{C}$ to the Eq. (10). The value of the exponent $\delta$ for TL-LSMO- 0.3 is larger than the $\delta$ value in 3D universality classes defined for the shortrange interaction, see Table 2 . These exponents $\beta, \gamma$ and $\delta$ for TL-LSMO- 0.3 should satisfy the Eq. $(15)^{47}$. The value $\delta=15.245$ is obtained using the value of $\beta$ and $\gamma$ determined from MAPS in Eq. (15). The $\delta$ value obtained from Eq. (15) is consistent with the $\delta$ value determined from the critical isotherm. Hence, both the exponents $\beta$ and $\gamma$ are found to satisfy the Widom-scaling relation defined in Eq. (15).

Further, the exponents $\beta$ and $\gamma$ have been determined more accurately by Kouvel-Fisher (KF) method ${ }^{48}$. The spontaneous magnetization $\left[\mathrm{M}_{S}(\mathrm{~T})\right]$ and inverse magnetic susceptibility $\left[\chi_{0}(\mathrm{~T})\right]$ are determined from 

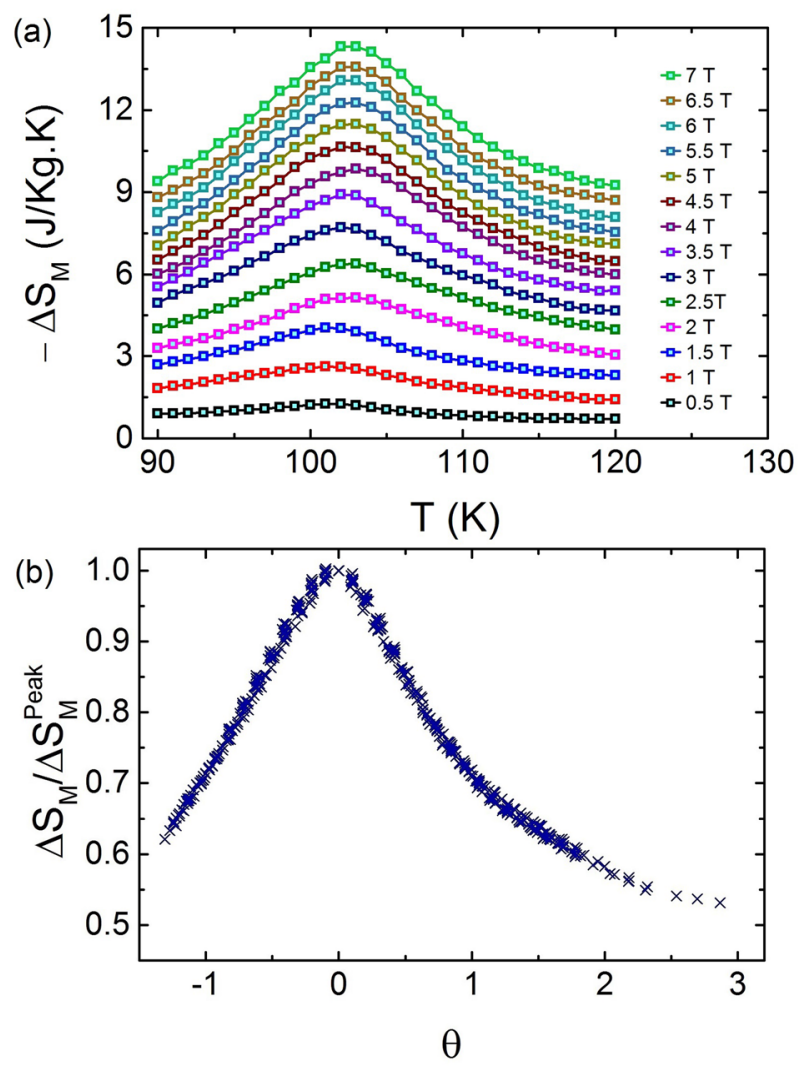

Figure 3. (a) The evolution of $\Delta S_{M}$ vs. $\mathrm{T}$ at different fields $(0.5 \rightarrow 7 \mathrm{~T})$ determined by M-H curve [Fig. 2(b)], which shows a continuous nonmonotonic change of $\Delta S_{M}$ around $\mathrm{T}_{C}$. (b) Universal curve for TL-LSMO-0.3 shows the collapse of all $\Delta S_{M}$ on single curve, which is the characteristic of second-order phase transition. (Figures have been drawn using OriginPro 2015 software).

the intersection with the axes $\mathrm{M}^{1 / \beta}$ and $\mu_{0} \mathrm{H} / \mathrm{M}^{1 / \gamma}$, respectively. The intercepts are obtained from the linear extrapolation in the MAPs plotted for the $2 \mathrm{D}$ short-range Ising model because of the nearly parallel behavior of the isotherms, as displayed in Fig. 6. The variation of $\mathrm{M}_{S}$ and $\chi_{0}$ with temperature for TL-LSMO-0.3 is shown in Fig. 7a. The solid lines in Fig. 7a represent fit to the $\mathrm{M}_{S}$ and $\chi_{0}$ using Eqs. (8) and (9), respectively. The KF method has the following form, which is obtained by Eq. (11) in the limit $\mathrm{H} \rightarrow 0$ for $\mathrm{T}<\mathrm{T}_{C}$ and $\mathrm{T}>\mathrm{T}_{C}$

$$
\frac{M_{S}(T)}{d M_{S}(T) / d T}=\frac{T-T_{C}}{\beta}
$$

and

$$
\frac{\chi_{0}^{-1}(T)}{d \chi_{0}^{-1}(T) / d(T)}=\frac{T-T_{C}}{\gamma} .
$$

The value of exponents $\beta$ and $\gamma$ can be determined from the slopes $1 / \beta$ and $1 / \gamma$ obtained from the linear variation of $M_{S}\left(d M_{S}(T) / d T\right)^{-1}$ vs. T and $\chi_{0}^{-1}\left(d \chi_{0}^{-1} / d T\right)^{-1}$ vs. T, respectively. The intersection with the temperature axis yields $\mathrm{T}_{C}$, as shown in Fig. $7 \mathrm{~b}$. Solid lines in Fig. $7 \mathrm{~b}$ represent the fit to the $M_{S}\left(d M_{S}(T) / d T\right)^{-1}$ vs. T and $\chi_{0}^{-1}\left(d \chi_{0}^{-1} / d T\right)^{-1}$ vs. T using Eqs. (3) and (4), respectively. The KF method results the value of exponents $\beta$ $=0.120 \pm 0.003$ with $\mathrm{T}_{C}=103.24 \pm 0.01 \mathrm{~K}$ and $\gamma=1.710 \pm 0.005$ with $\mathrm{T}_{C}=103.12 \mathrm{~K} \pm 0.02$. These results are consistent with the value of exponents obtained from the MAPs.

Further, we confirm that the obtained exponents are not different above and below $\mathrm{T}_{C}$. As we know, one can also deduce the critical exponents of a magnetic sample using scaling theory, which states that for an appropriate value of the critical exponents $(\beta$ and $\gamma)$, the plot of scaled magnetization $\left(\mathrm{m}=\mathrm{M} \epsilon^{-\beta}\right)$ vs. renormalized field (h $\left.=\mu_{0} \mathrm{H} \epsilon^{-(\beta+\gamma)}\right)$ should fall onto two separate curves: one for $\mathrm{T}<\mathrm{T}_{C}$ and other for $\mathrm{T}>\mathrm{T}_{C}$. Figure 8 represents the $\mathrm{M} \epsilon^{-\beta}$ as a function of $\mu_{0} \mathrm{H} \epsilon^{-(\beta+\gamma)}$ below and above $\mathrm{T}_{C}$ in TL-LSMO-0.3. One can see that all the magnetization curve fall onto two curves below and above $\mathrm{T}_{C}$ separately, when the value of $\mathrm{T}_{C}$ and exponents are chosen as $\mathrm{T}_{C}$ $=103.17 \pm 0.01 \mathrm{~K}, \beta=0.121 \pm 0.001, \gamma^{\prime}=1.710 \pm 0.005$ for $\mathrm{T}<\mathrm{T}_{C}$ and $\gamma=1.702 \pm 0.002$ for $\mathrm{T}>\mathrm{T}_{C}$ in Eq. (12). Further, we have plotted $\mathrm{m}^{2} \mathrm{vs} . \mathrm{h} / \mathrm{m}$ and again found that all the data collapse onto two separate curves above and below $\mathrm{T}_{C}$, respectively. This confirms that the critical exponents are reliable, unambiguous and the interactions get renormalized appropriately following the scaling equation of state in the critical regime. 

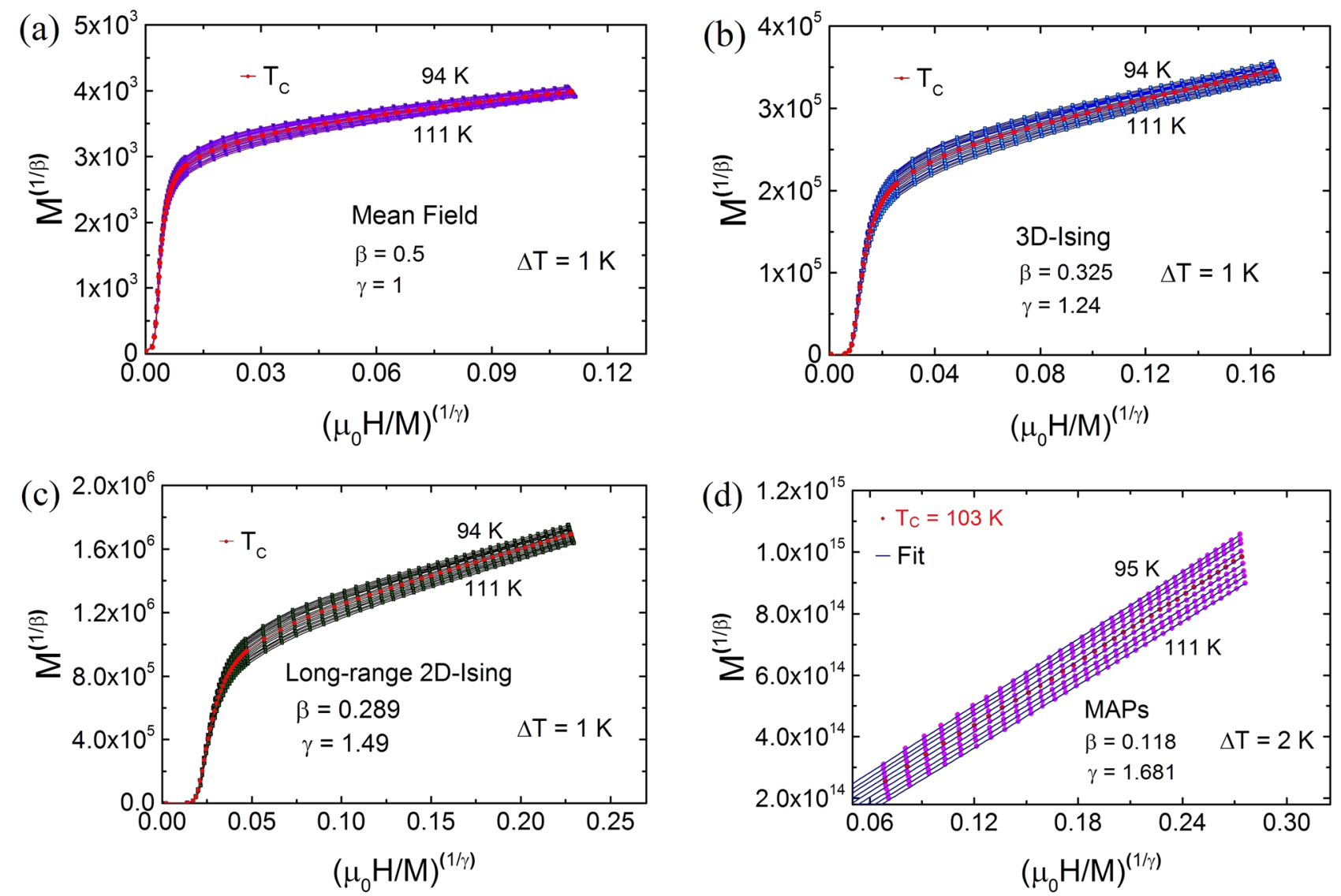

Figure 4. Isotherms of $\mathrm{M}^{2}$ vs. $\mu_{0} \mathrm{H} / \mathrm{M}$ for $94 \mathrm{~K} \leq \mathrm{T} \leq 111 \mathrm{~K}(|\epsilon| \leq 0.1)$ with (a) the Arrott plot (mean-field theory), which shows non-linear or non-mean-field type behavior even in higher magnetic field with positive slopes corresponding to the second-order phase transition. (b) MAPs for 3D-Ising with short-range interaction (c) MAPs for 2D-Ising model with Long-range interaction and (d) MAPs of M-H curves with $\beta=0.118$ and $\gamma=1.681$ in TL-LSMO-0.3. Solid lines are corresponding to the fits by the Eq. (11). (Figures have been drawn using OriginPro 2015 software).

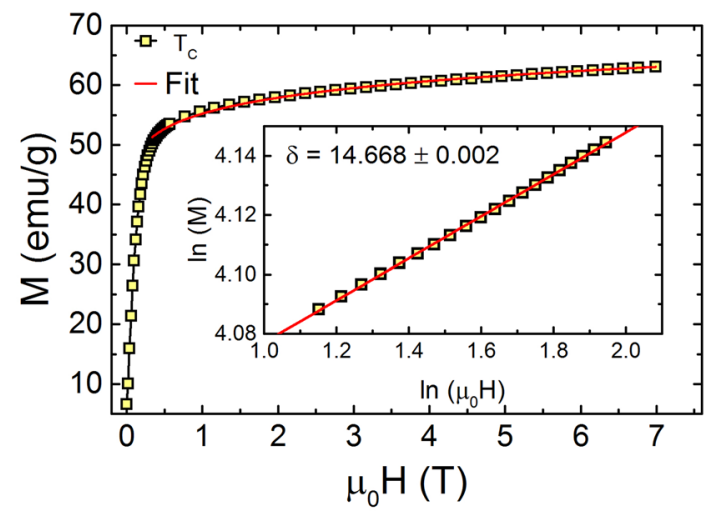

Figure 5. $\mathrm{M}-\mathrm{H}$ curve at $\mathrm{T}_{C}=103 \mathrm{~K}$ and inset shows log-log plot of the curve. Solid line represents the fit to the Eq. (10) and yields $\delta=14.668 \pm 0.002$ mentioned in graph is obtained from the fit to the Eq. (10). (The figure has been drawn using OriginPro 2015 software).

\section{Spin interaction}

Finally, we discuss the range and dimensionality of the TL-LSMO-0.3 with the help of the renormalization group theory. For a homogeneous magnet, the universality class of the magnetic phase transition is defined by the interaction $\mathrm{J}(\mathrm{r})$. Fisher et al. ${ }^{52}$ used renormalization group theory and suggested that the exchange interaction decays with distance $\mathrm{r}$ as $\mathrm{J}(\mathrm{r}) \sim r^{-(d+\sigma)}$, where $\mathrm{d}$ is space dimensionality and $\sigma$ is the range of the interaction. 


\begin{tabular}{|c|c|c|c|c|c|}
\hline & Method & $T_{C}(\mathbf{K})$ & $\beta$ & $\gamma$ & $\delta$ \\
\hline \multicolumn{6}{|l|}{ Theory } \\
\hline Mean field ${ }^{49,50}$ & & & 0.5 & 1 & 3 \\
\hline Tricritical mean field 49,51 & & & 0.25 & 1 & 5 \\
\hline 3D-Ising $(\mathrm{d}=3, \mathrm{n}=1)^{49,50}$ & RG- $\phi^{4}$ & & 0.325 & 1.24 & 4.82 \\
\hline $3 \mathrm{D}-\mathrm{XY}(\mathrm{d}=3, \mathrm{n}=2)^{49,50}$ & RG- $\phi^{4}$ & & 0.346 & 1.316 & 4.81 \\
\hline 3D-Heisenberg $(d=3, n=3)^{49,50}$ & RG- $\phi^{4}$ & & 0.365 & 1.386 & 4.8 \\
\hline Short-range 2D-Ising ${ }^{44,52}$ & Onsager solution & & 0.125 & 1.75 & 15 \\
\hline Long-range 2D-Ising ${ }^{52}$ & RG- $\epsilon^{\prime}$ & & 0.289 & 1.49 & 6 \\
\hline \multicolumn{6}{|l|}{ Experiment } \\
\hline \multirow{6}{*}{$\mathrm{La}_{2.1} \mathrm{Sr}_{1.9} \mathrm{Mn}_{3} \mathrm{O}_{10}$} & MAPs & $103.54 \pm 0.03$ & $0.118 \pm 0.004$ & $1.681 \pm 0.006$ & \\
\hline & $\mathrm{CI}$ & 103 & & & $14.668 \pm 0.002$ \\
\hline & KF & $103.24 \pm 0.01$ & $0.120 \pm 0.003$ & & \\
\hline & & $103.12 \pm 0.02$ & & $1.710 \pm 0.005$ & \\
\hline & RG & & 0.145 & 1.91 & 14.172 \\
\hline & Scaling & $103.17 \pm 0.01$ & $0.121 \pm 0.001$ & $1.710 \pm 0.005$ & \\
\hline
\end{tabular}

Table 2. Comparison of critical exponents $\beta, \gamma$ and $\delta$ of TL-LSMO-0.3 with various theoretical models for three dimension and two dimension. RG: Renormalization group, CI: Critical isotherm.

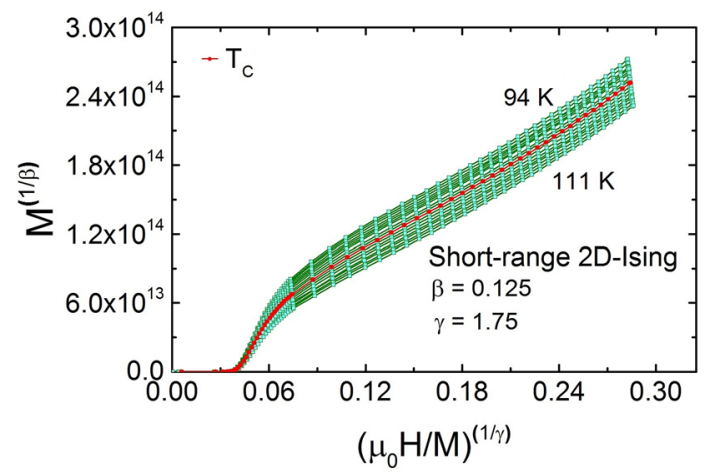

Figure 6. Isotherms of $\mathrm{M}^{2}$ vs. $\mu_{0} \mathrm{H} / \mathrm{M}$ for $94 \mathrm{~K} \leq \mathrm{T} \leq 111 \mathrm{~K}(|\epsilon| \leq 0.1)$ with the short-range 2D-Ising model. (The figure has been drawn using OriginPro 2015 software).

Also, they have discussed the validity of such a model for $\sigma<2$ having long-range interactions. Further, the critical exponent $\gamma$ associated with the susceptibility can be given as

$$
\gamma=1+\frac{4}{d}\left(\frac{n+2}{n+8}\right) \Delta \sigma+\left(\frac{8(n+2)(n-4)}{d^{2}(n+8)^{2}}\right) \times\left[1+\frac{2 G\left(\frac{d}{2}\right)(7 n+20)}{(n-4)(n+8)}\right] \Delta \sigma^{2},
$$

where $\Delta \sigma=\left(\sigma-\frac{d}{2}\right)$ and $G\left(\frac{d}{2}\right)=3-\frac{1}{4}\left(\frac{d}{2}\right)^{2}, n$ is spin dimensionality. The range of interaction $\sigma$ and dimensionality of both space and spin are determined by the iterative method ${ }^{53}$ in which the value of $\sigma$ is chosen for a particular set of $\{d: n\}$ such that the Eq. (5) results in the value of exponent $\gamma$ close to the experimentally determined, $\gamma=1.71$. This process is repeated for the different sets of $\{d: n\}=\{2,3: 1,2,3\}$ to ensure the space and spin dimensionality of the TL-LSMO-0.3. Further, the remaining exponents can be determined with the help of Eqs. (14), (15) and $\sigma$ value using following expressions: $\alpha=2-\nu \mathrm{d}, \nu=\gamma / \sigma, \gamma=\nu(2-\eta)$ and $\eta=2-\sigma$. We found that $\{d: n\}=\{2: 1\}$ yields a value $\sigma=1.69$, which results in the $\gamma$ value close to the experimentally determined value of $\gamma$ for TL-LSMO-0.3. The value of $\sigma=1.69$ is then used to determine the remaining exponents, such as; $\beta=$ $0.135, \gamma=1.91$ and $\delta=14.172$, which are close to the value of exponents obtained from previous methods MAPs, $\mathrm{KF}$ and scaling analysis (Table 2). We have also examined the remaining $3 \mathrm{D}$ and $2 \mathrm{D}$ models, but they cannot describe the experimental results obtained for TL-LSMO-0.3. For example, the 3D-Heisenberg $\{d: n\}=\{3: 3\}$, 3D-XY $\{d: n\}=\{3: 2\}$ and 3D-Ising models $\{d: n\}=\{3: 1\}$ with short-range exchange interaction yield the value of exponent $\gamma=1.25,1.27$ and 1.23, respectively. Similarly, the $2 \mathrm{D}$ Heisenberg $\{d: n\}=\{2: 3\}$ and the $2 \mathrm{D}$ $\mathrm{XY}\{d: n\}=\{2: 2\}$ models defined for the short-range exchange interaction yield $\gamma=2.56$ and 2.30 , respectively. The other calculated exponents $(\beta$ and $\delta$ ) by using respective $\sigma$ values for different models $\{d: n\}=\{2: 2,3\}$, $\{d: n\}=\{3: 1,2,3\}$ also show significant differences from experimental results for $\beta$ and $\delta$. Hence, all the other 2D and 3D models can be discarded. The long-range mean field model is valid for $\sigma \leq 3 / 2$ and J(r) decreases as 

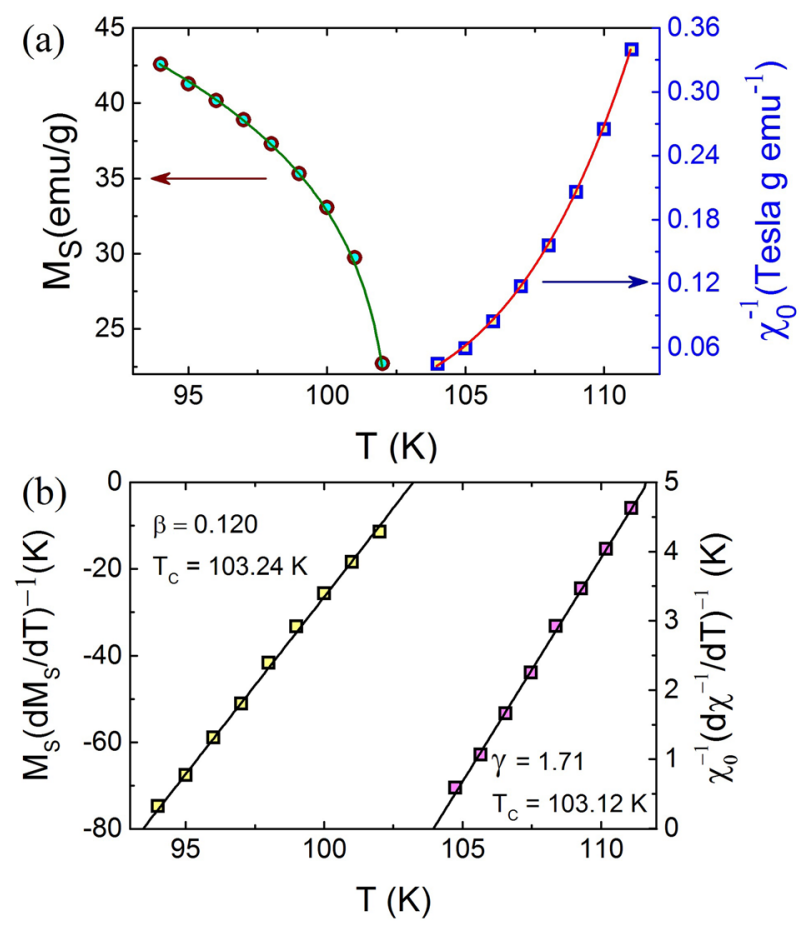

Figure 7. (a) Temperature variation of spontaneous magnetization $\mathrm{M}_{S}(\mathrm{~T}, 0)$ (left) and inverse susceptibility $\chi_{0}^{-1}$ (T, 0) (right). (b) KF plots for the $M_{S}\left(d M_{S}(T) / d T\right)^{-1}$ (left) and $\chi_{0}^{-1}\left(d \chi_{0}^{-1} / d T\right)^{-1}$ (right). (Figures have been drawn using OriginPro 2015 software).
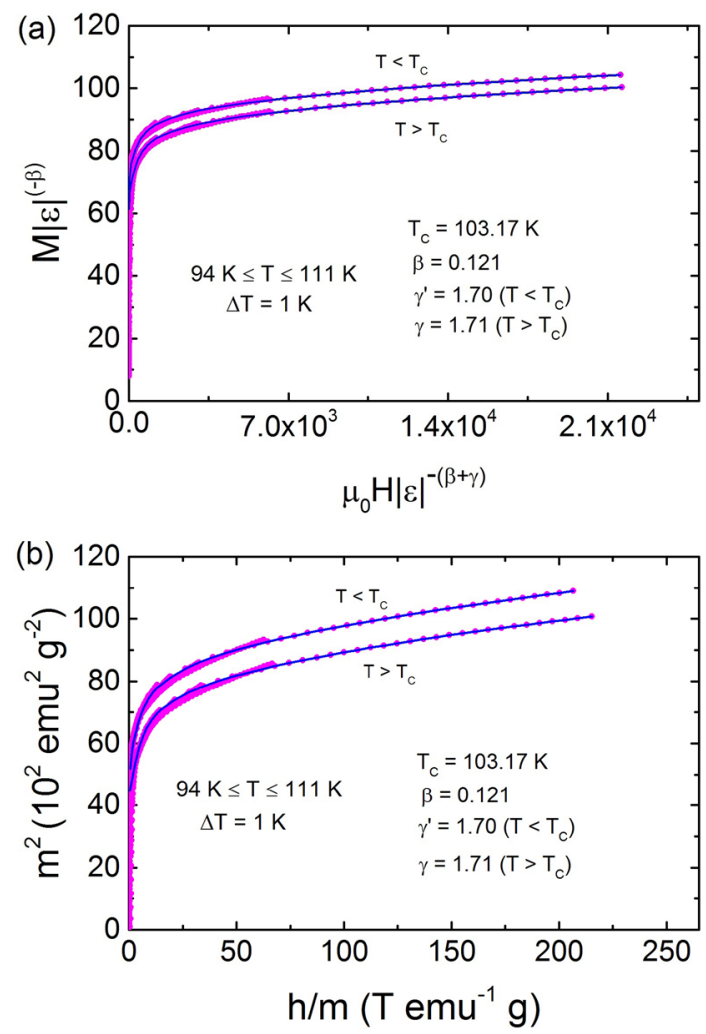

Figure 8. (a) Scaling of the M-H curve below and above $\mathrm{T}_{C}$ for TL-LSMO-0.3 in critical region $\epsilon \leq 0.1$ using Eq. (12). Solid lines show best fit polynomials. Collapse of all the $\mathrm{M}-\mathrm{H}$ curve on single curve below and above $\mathrm{T}_{C}$ separately confirms the validity of obtained exponents. $(\mathbf{b}) \mathrm{m}^{2} \mathrm{vs} \mathrm{h} / \mathrm{m}$ below and above $T_{C}$ also shows collapse of curve again validate the obtained results. (Figures have been drawn using OriginPro 2015 software). 


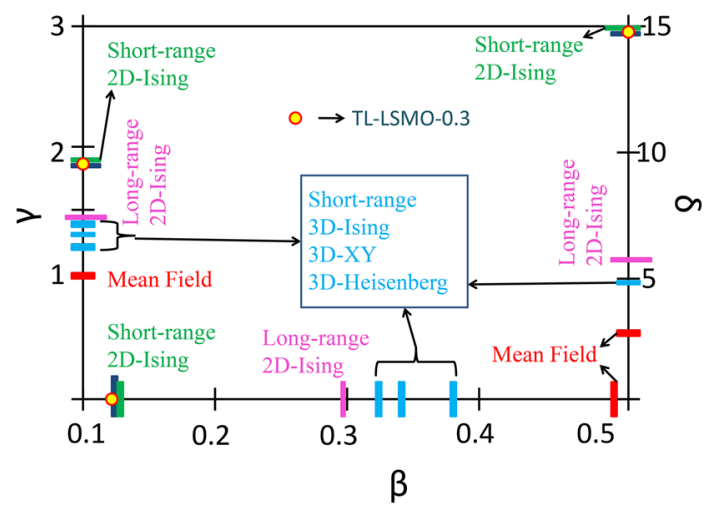

Figure 9. Comparison of different exponents of TL-LSMO-0.3 (denoted as closed circles filled with yellow color) with that of standard universality classes. Different vertical bars represents different models for shortrange and long-range exchange interactions. Short-range $\rightarrow$ the 2D-Ising (green bar), 3D-Ising (sky blue bar), $3 \mathrm{D}$-XY (sky blue bar) and 3D-Heisenberg (sky blue bar) and long-range $\rightarrow 2 \mathrm{D}$-Ising model (pink bar). (The figure has been drawn using microsoft office powerpoint software).

$\mathrm{J}(\mathrm{r}) \sim \mathrm{r}^{-4.5}$. For $\sigma \geq 2$, only the short-range 3D-Heisenberg model is valid and $\mathrm{J}(\mathrm{r})$ varies as $\mathrm{J}(\mathrm{r}) \sim \mathrm{r}^{-5}$. The other $3 \mathrm{D}$ universality classes for short-range lies between $3 / 2<\sigma<2$, where $\mathrm{J}(\mathrm{r})$ decreases as $\mathrm{J}(\mathrm{r}) \sim \mathrm{r}^{-\dot{d}-\sigma}$. All the theoretical models with short-range exchange interaction vary with distance $\mathrm{r}$ as $\mathrm{J}(\mathrm{r}) \sim \mathrm{e}^{-(r / b)}$ (where b is correlation length). The renormalization group analysis suggests that the spin interaction in TL-LSMO-0.3 is of a short-range $2 \mathrm{D}$ Ising $\{d: n\}=\{2: 1\}$ type with $\sigma=1.69$ and decays as $\sim \mathrm{r}^{-3.69}$.

\section{Discussion}

All the findings in the above sections for TL-LSMO-0.3 yield the value of critical exponents close to the shortrange 2D-Ising model. A graphical comparison of the critical exponents $\beta, \gamma$ and $\delta$ for TL-LSMO-0.3 with the various theoretical models is represented in Fig. 9. The obtained results of the exponents consistent with the Q2D-layered structural characteristic of TL-LSMO-0.3 and emphasize that the magnetic anisotropy is playing a crucial role in the magnetism of the TL-LSMO-0.3. The 2D magnetism in TL-LSMO-0.3 also emphasizes that the inter-layer interaction is weakened around $\mathrm{T}_{C}$. In contrast, the intra-layer interaction becomes stronger, which leads to a 2D FM in TL-LSMO-0.3. Our results for TL-LSMO-0.3 are consistent with the Taroni et al. ${ }^{54}$ criterion, according to which the value of critical exponent $\beta$ for $2 \mathrm{D}$ magnets should lie in the $0.1 \geq \beta \geq 0.25$. Similar results have been reported in bi-layer $\mathrm{La}_{2-2 x} \mathrm{Sr}_{1+2 x} \mathrm{Mn}_{2} \mathrm{O}_{7}$ in which short-range 2D FM ordering occurs around $\mathrm{T}_{C}{ }^{55,56}$. Osborn et al. ${ }^{55}$ performed neutron scattering measurement in bi-layer $\mathrm{La}_{2-2 x} \mathrm{Sr}_{1+2 x} \mathrm{Mn}_{2} \mathrm{O}_{7}$ for $\mathrm{x}$ $=0.4$ and claimed that there is a short-range $2 \mathrm{D}$-Ising interaction with $\beta=0.13 \pm 0.01$. Gordon et al. ${ }^{56}$ performed specific heat measurement on $\mathrm{La}_{2-2 x} \mathrm{Sr}_{1+2 x} \mathrm{Mn}_{2} \mathrm{O}_{7}$ for $\mathrm{x}=0.4$ and claimed that the obtained result is consistent with $2 \mathrm{D}-\mathrm{XY}$ or $2 \mathrm{D}$-Ising critical fluctuation. There is no neutron diffraction data on the tri-layer $\mathrm{La}_{3}-3 x \mathrm{Sr}_{1+3 x}$ $\mathrm{Mn}_{3} \mathrm{O}_{10}$. However, one can get an idea about the spin structure and spin-spin interaction from the neutron diffraction data for bi-layer manganites.

Next, we discuss the unconventional behavior of temperature-dependent magnetization and magnetic spin structure in different regions. Conventionally, when a FM material undergoes a magnetic phase transition from FM to PM state, the magnetic moment of the system becomes zero above $\mathrm{T}_{C}$. As shown in Fig. 2a, the magnetic moment of the FC curve for TL-LSMO- 0.3 is non-zero above $\mathrm{T}_{C}$ and also another transition appears at a higher temperature $\approx 263 \mathrm{~K}$, denoted as $\mathrm{T}^{*}$. The non-zero magnetization above $\mathrm{T}_{C}$ emphasizes that the phase transition in TL-LSMO-0.3 is not a FM to PM state.A similar magnetic phase transition has been observed in bi-layer $\mathrm{La}_{2-2 x} \mathrm{Sr}_{1+2 x} \mathrm{Mn}_{2} \mathrm{O}_{7} \mathrm{RP}$ series manganites and extensive studies have been conducted to explore the magnetic structure of this region (between $\mathrm{T}_{C}$ and $\mathrm{T}^{*}$ ). Kimura et al. ${ }^{17}$ studied $\mathrm{La}_{1.4} \mathrm{Sr}_{1.6} \mathrm{Mn}_{2} \mathrm{O}_{7}$ and claimed that there is a short-range $2 \mathrm{D} \mathrm{FM}$ ordering between $\mathrm{T}_{C}$ and $\mathrm{T}^{*}$, which gives rise to the finite magnetic moment in this region and above $\mathrm{T}^{*}$ system goes to the $\mathrm{PM}$ state. The disagreement of $2 \mathrm{D} \mathrm{FM}$ characteristic above $\mathrm{T}_{C}$ was shown by Heffner et al. ${ }^{58}$ from the muon spin rotation study in bi-layer $\mathrm{La}_{1.4} \mathrm{Sr}_{1.6} \mathrm{Mn}_{2} \mathrm{O}_{7}$ and claimed that there is no evidence of $2 \mathrm{D}$ magnetic ordering above $\mathrm{T}_{C}$. Later, a rigorous neutron scattering study in bi-layer $\mathrm{La}_{2}-2 x \mathrm{Sr}_{1+2 x}$ $\mathrm{Mn}_{2} \mathrm{O}_{7}$ for $\mathrm{x}=0.4$ by Osborn et al. ${ }^{55}$ revealed that there is a strong canting of the $\mathrm{Mn}$ spins in adjacent $\mathrm{MnO}_{2}$ layers within each $\mathrm{MnO}_{2}$ bi-layer above $\mathrm{T}_{C}$ and the canting angle depends on both temperature and magnetic field. Their neutron scattering study revealed that the FM and antiferromagnetic (AFM) magnetic ordering are inhomogeneously distributed in approximately equal volume above $\mathrm{T}_{C}$. Therefore, a non-collinear spin correlation or the canting of spins arises due to the competing FM DE and AFM superexchange (SE) interaction. The intra-bi-layer or inter-planer interaction is substantially weaker than the intra-planar interaction, which produces a large magnetic anisotropy in the exchange interactions. The FM in manganites is governed by the DE mechanism based on the hopping of the electrons and the kinetic energy of mobile electrons is lowered by polarizing the $\mathrm{Mn}$ spins, which are localized in the $\mathrm{MnO}_{2}$ plane. Hence, there would be larger free energy within the plane due to the energy acquired from delocalized electrons ${ }^{55}$. A comparatively large number of Mn spins can take part 


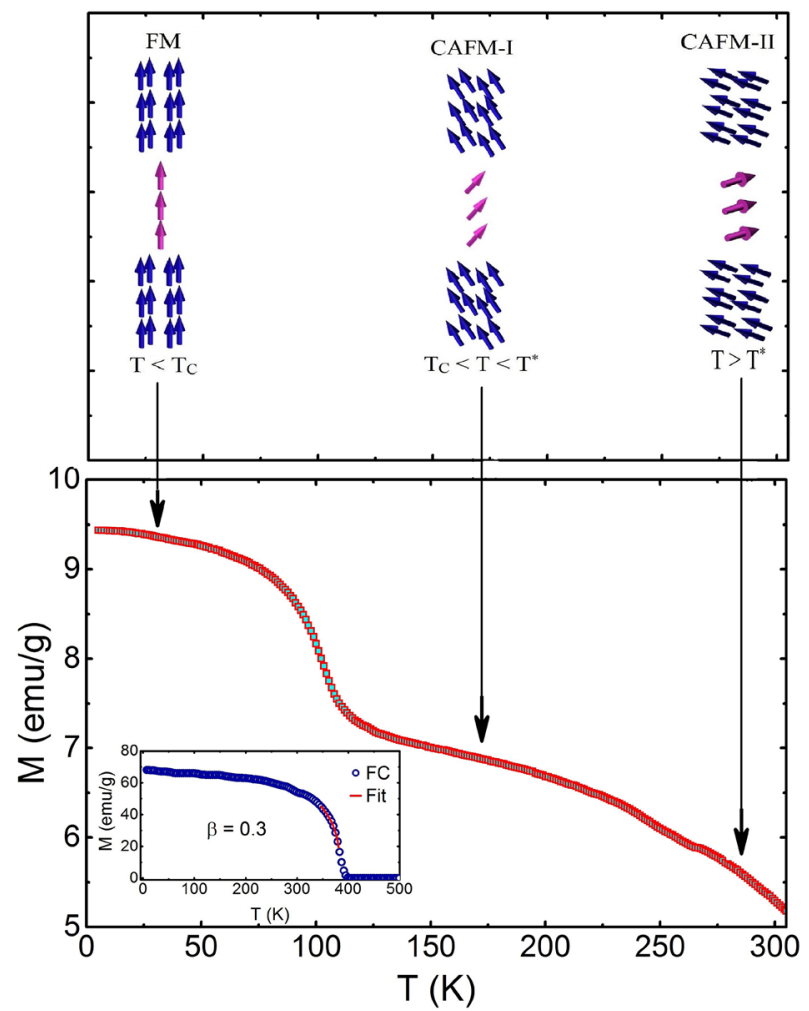

Figure 10. Spin structure in the different temperature range of TL-LSMO-0.3. Below $\mathrm{T}_{C}$, i.e., $\mathrm{T}<\mathrm{T}_{C}$ the spins have FM alignment. First type of canting of spins occur in the range $\mathrm{T}_{C}<\mathrm{T}<\mathrm{T}^{*}$ and above $\mathrm{T}^{*}$ second type of canting is represented. The inset shows the FC curve for infinite-layer $\mathrm{La}_{0.7} \mathrm{Sr}_{0.3} \mathrm{MnO}_{3}{ }^{57}$. The solid line (red color) represents the fit to the Eq. (8) and yield the value of exponent $\beta=0.3$. (Figures have been drawn using OriginPro 2015 software).

in the FM cluster than among the adjacent planes, where only two Mn spin sites are available. Therefore, the SE interaction strongly affects the spin interactions along the c-axis compared to the spins in the ab-plane ${ }^{55}$. This is the reason why intra-planar interaction is stronger than intra-bi-layer interaction.

We have not performed any local probe measurements to determine the spin structure in different temperature ranges of TL-LSMO-0.3. Figure 10 shows the possible magnetic structures above and below $\mathrm{T}_{C}$ in TL-LSMO-0.3. Different magnetic phases shown in Fig. 10 are purely speculative and we have obtained these phases based on the neutron diffraction measurement in bi-layer $\mathrm{La}_{2-2 x} \mathrm{Sr}_{1+2 x} \mathrm{Mn}_{2} \mathrm{O}_{7}$ manganite ${ }^{55,59}$, which shows a similar magnetic behavior with temperature. The inset of Fig. 10 shows the FC curve for infinite-layer $\mathrm{La}_{0.7} \mathrm{Sr}_{0.3} \mathrm{MnO}_{3}{ }^{57}$. Based on the above discussions for non-zero magnetization in bi-layer $\mathrm{La}_{2-2 x} \mathrm{Sr}_{1+2 x} \mathrm{Mn}_{2} \mathrm{O}_{7}$, we propose that a similar inhomogeneous distribution of FM and AFM clusters are giving rise the canted AFM-I (CAFM-I) type spin structure above $\mathrm{T}_{C}$, which is responsible for the non-zero magnetization in TL-LSMO-0.3. The magnetic moment of the system is continuously decreasing with an increase in temperature and the system undergoes another transition at $\mathrm{T}^{*}$. It is noted that the magnetic moment is still non-zero above second transition $\mathrm{T}^{*}$, which indicate that the system is going to another canted AFM-II (CAFM-II) state with a canting angle greater than that of the CAFM-I state and responsible for a finite magnetic moment above the second transition $\mathrm{T}^{*}$. The layered crystal structure of the TL-LSMO- 0.3 suggests that there are different types of interactions present in the system; inter-tri-layer $\left(J^{\prime}\right)$, inter-layer, or intra-tri-layer $\left(J_{c}\right)$ and intra-planer $\left(J_{a b}\right)$ interaction ${ }^{60}$. The two manganese ions $\mathrm{Mn}^{3+}$ and $\mathrm{Mn}^{4+}$ are distributed in the ratio of $\mathrm{Mn}^{3+}: \mathrm{Mn}^{4+}$ 2.33:1 in the system. Depending upon the distribution and distance between $\mathrm{Mn}$ spins in different directions, the order of the strength of interaction can be given as $J_{a b}>J_{c}>>J^{\prime 60}$. The intra-planer interaction $J_{a b}$ is the DE interaction, i.e., the spins are coupled ferromagnetically and the intra-tri-layer interaction $J_{c}$ is SE interaction, which implies that the spins are coupled antiferromagnetically. On the other hand, the inter-tri-layer interaction $J^{\prime}$ is the direct exchange interaction. Although the DE interaction is strong in ab-plane, but a minor AFM interaction also coexists. Similarly, the intra-tri-layer interaction $J_{c}$ is an SE interaction along the c-axis, but a weak DE interaction can also coexist. The intra-planer interaction $J_{a b}$ is the strongest and dominates over other interactions $\left(J_{c}\right.$ and $\left.J^{\prime}\right)$. $J_{a b}$ combined with the anisotropy give rise to the $2 \mathrm{D}$-Ising like spin structure below $\mathrm{T}_{C}$. The solid line (red color) represents the fit to the Eq. 8 and yield the value of exponent $\beta=0.3$, which is close to the $3 \mathrm{D}$-Ising universality class. Michael et al. ${ }^{61}$ and Vasiliu et al. ${ }^{62}$ performed neutron scattering in infinite-layer $\mathrm{La}_{0.7} \mathrm{Sr}_{0.3} \mathrm{MnO}_{3}$ and shown that it belongs to the short-range 3D-Ising universality class with $\beta=0.295$ and 0.3 , respectively. One can see that the magnetic moment of infinite-layer $\mathrm{La}_{0.7} \mathrm{Sr}_{0.3} \mathrm{MnO}_{3}$ above $\mathrm{T}_{C}$ is zero in contrast to TL-LSMO-0.3. With reduced dimensionality from $3 \mathrm{D}$ to $\mathrm{Q} 2 \mathrm{D}$, the system changes from 3D-Ising to $2 \mathrm{D}$-Ising like spin-spin interaction and there 
exists a canted AFM magnetic structure between FM and PM state due to the existence of different exchange interactions. It is well known that the different interactions are responsible for different spin structures. The exchange interaction aligns the spins parallel to each other. In contrast, long-range dipolar interaction favors a close loop of spins and anisotropy energy favors perpendicular alignment of spins to the plane. Hence, the anisotropy in a magnetic system results in the Ising spin structure and the system behaves as a uniaxial magnet. The Ising interaction below $\mathrm{T}_{C}$ in TL-LSMO-0.3 emphasizes that magnetic anisotropy plays a crucial role in the magnetism of the TL-LSMO-0.3. It is believed that the skyrmions in manganite perovskites result from the competition between different energies such as exchange interaction, long-range dipolar interaction and anisotropy energy. Keeping in view of the observation of bi-skyrmion in the bi-layer $\mathrm{La}_{1.37} \mathrm{Sr}_{1.63} \mathrm{Mn}_{2} \mathrm{O}_{7}$, which has similar magnetic properties to the TL-LSMO-0.3, we contemplate that TL-LSMO-0.3 should also host the skyrmions.

All the above discussions and experimental observations imply that much more experimental and theoretical works are needed to thoroughly understand the magnetism in tri-layer $\mathrm{La}_{3}-3 x \mathrm{Sr}_{1+3 x} \mathrm{Mn}_{3} \mathrm{O}_{10}$ manganite perovskite. The magnetic and transport properties of tri-layer $\mathrm{La}_{3-3 x} \mathrm{Sr}_{1+3 x} \mathrm{Mn}_{3} \mathrm{O}_{10}$ manganite perovskite for different Sr concentration is not yet explored. Therefore, it is highly desirable to establish the structural, magnetic and electronic phase diagram of tri-layer $\mathrm{La}_{3}-3 x \mathrm{Sr}_{1+3 x} \mathrm{Mn}_{3} \mathrm{O}_{10}$ because it may be a potential candidate for future spintronics. We hope the present study will prompt further investigation in understanding the magnetic phase transition and different types of exchange interaction in the low dimensional RP series manganite perovskites.

\section{Conclusion}

In summary, we have established an understanding of the phase transition in a novel quasi-two-dimensional ferromagnetic tri-layer $\mathrm{La}_{2.1} \mathrm{Sr}_{1.9} \mathrm{Mn}_{3} \mathrm{O}_{10} \mathrm{RP}$ series manganite. We have discussed the low dimensionality in the magnetic properties of the tri-layer $\mathrm{La}_{2.1} \mathrm{Sr}_{1.9} \mathrm{Mn}_{3} \mathrm{O}_{10}$ manganite perovskite. The collapse of all the temperature dependent magnetic entropy change curves on a single universal curve confirms the second-order phase transition in $\mathrm{La}_{2.1} \mathrm{Sr}_{1.9} \mathrm{Mn}_{3} \mathrm{O}_{10}$. A comprehensive experimental study of the critical properties is performed using isothermal magnetization in the vicinity of the Curie temperature $\mathrm{T}_{C}$. We have used various techniques, including the modified Arrott plots (MAPs), Kouvel-Fisher (KF) method, scaling and critical isotherm analysis to determine the critical exponents of the $\mathrm{La}_{2.1} \mathrm{Sr}_{1.9} \mathrm{Mn}_{3} \mathrm{O}_{10}$. The obtained critical exponents for $\mathrm{La}_{2.1} \mathrm{Sr}_{1.9} \mathrm{Mn}_{3}$ $\mathrm{O}_{10}$ are close to theoretical values compatible with the $2 \mathrm{D}$-Ising model with short-range interaction. The critical exponents of the $\mathrm{La}_{2.1} \mathrm{Sr}_{1.9} \mathrm{Mn}_{3} \mathrm{O}_{10}$ were also determined using the renormalization group approach for a twodimensional (2D) Ising system with short-range interactions decaying as $\mathrm{J}(\mathrm{r}) \sim \mathrm{r}^{-d-\sigma}$ with $\sigma=1.69$. We suggest that the strong anisotropy and layered structure are playing a crucial role in resulting in the Ising-like interaction in $\mathrm{La}_{2.1} \mathrm{Sr}_{1.9} \mathrm{Mn}_{3} \mathrm{O}_{10}$. Based on results obtained for $\mathrm{La}_{2.1} \mathrm{Sr}_{1.9} \mathrm{Mn}_{3} \mathrm{O}_{10}$ in the present study, we propose that the $\mathrm{La}_{3}-3 x \mathrm{Sr}_{1+3 x} \mathrm{Mn}_{3} \mathrm{O}_{10}$ can be a potential candidate for the skyrmion host material. Finally, we propose that the non-zero magnetic moment above $\mathrm{T}_{C}$ is due to the canted antiferromagnetic spin orientation.

\section{Appendix A: Theoretical background and methodology}

The non-equilibrium dynamics of the magnetic systems near the magnetic phase transition temperature $\mathrm{T}_{C}$ have received increasing attention ${ }^{63-69}$. At time $t=0$, the system is quenched in the vicinity of the $\mathrm{T}_{C}$ from an equilibrium state away from the $\mathrm{T}_{C}$. This results in a critical slow down due to the slow relaxation towards the new equilibrium state of the system driven by the sudden quenching in the vicinity of $\mathrm{T}_{C}$. Generally, the Langevintype equation is used to define the theoretical models for the critical dynamics of a system, which is governed by the Ginzburg-Landau theory for conserved or non-conserved order parameters ${ }^{65-69}$. Different universality classes are deduced from different theoretical models, which depend on the associated conservation laws and model parameters $\mathrm{n}$ and $\mathrm{d}$. The critical analysis of magnetic systems gives rise the vital information such as the universality class, behavior of phase transition and spin interaction of the system.

According to the Landau (mean-field) theory, the magnetic free energy $\mathrm{F}_{M}$ of a second-order magnetic system can be expressed as a power series in the order parameter $M$ in the vicinity of the $T_{C}$ as

$$
\begin{aligned}
F(M, T)= & F(0)+\frac{a(T)}{2} M^{2}+\frac{b(T)}{4} M^{4} \\
& +\cdots-\mu_{0} H M,
\end{aligned}
$$

where $a(T)$ and $b(T)$ are Landau coefficients. The equilibrium condition is defined by the minimization of the $\mathrm{F}_{M}$ as $\partial \mathrm{F}(\mathrm{M}, \mathrm{T}) / \partial \mathrm{M}=0$. The minimization of the $\mathrm{F}_{M}$ gives the following equation of state for a magnetic system near $\mathrm{T}_{C}$

$$
\mu_{0} H=a(T) M+b(T) M^{3} .
$$

This mean field approach fails in a critical region around $\mathrm{T}_{C}$ characterized by the Ginzburg criterion ${ }^{70}$. The critical behavior of a magnetic system which undergoes a second-order phase transition can be investigated in detail by a series of correlated critical exponents ${ }^{71}$. The divergence of correlation length $\xi=\xi_{0}\left|\left(T-T_{C}\right) / T_{C}\right|^{-v}(v-$ critical exponent) results in the universal scaling laws for $\mathrm{M}_{S}$ and $\chi_{0}(\mathrm{~T})$ in the vicinity of the second-order phase transition $\mathrm{T}_{C}$. The $\mathrm{M}_{S}(\mathrm{~T})$ is defined for $\mathrm{T}<\mathrm{T}_{C}$ and characterized by the exponent $\beta$. The $\chi_{0}(\mathrm{~T})$ is defined for $\mathrm{T}$ $>\mathrm{T}_{C}$ and characterized by the exponent $\gamma$. The isothermal magnetization $(\mathrm{M}-\mathrm{H})$ at $\mathrm{T}_{\mathrm{C}}$ is characterized by the exponent $\delta$. The $\mathrm{M}_{S}(\mathrm{~T})$ and $\chi_{0}(\mathrm{~T})$ show a power law dependence on the reduced temperature $\epsilon$ and the critical magnetization depends on $\mu_{0} \mathrm{H}^{71-73}$. The critical exponents before and after $\mathrm{T}_{C}$ can be given by

$$
M_{S}(T) \propto(-\epsilon)^{\beta} ; \epsilon<0, T<T_{C},
$$




$$
\chi_{0}^{-1}(T) \propto(\epsilon)^{\gamma} ; \epsilon>0, T>T_{C}
$$

and

$$
M \propto\left(\mu_{0} H\right)^{1 / \delta} ; \epsilon=0, T=T_{C} .
$$

Generally, the critical exponents associated with the $\mathrm{M}_{S}$ and $\chi_{0}(\mathrm{~T})$ should follow the Arrott-Noakes equation of state $^{46}$ in the asymptotic region $|\epsilon|<0.1$

$$
\left(\mu_{0} H / M\right)^{1 / \gamma}=a \epsilon+b M^{1 / \beta},
$$

where $\mathrm{a}$ and $\mathrm{b}$ are the material constant. Using scaling hypothesis, the magnetic equation of state, i.e., the relationship among the variables $\mathrm{M}\left(\mu_{0} \mathrm{H}, \epsilon\right), \mu_{0} \mathrm{H}$ and $\mathrm{T}$ in the asymptotic critical region is expressed as $\mathrm{s}^{73,74}$

$$
M\left(\mu_{0} H, \epsilon\right)=\epsilon^{\beta} f_{ \pm}\left(\mu_{0} H / \epsilon^{(\beta+\gamma)}\right),
$$

where $f_{+}$is defined for $T>T_{C}$ and $f_{-}$is for $T<T_{C}$. The magnetic equation of the state emphasizes that if the choice of the values of the critical exponents is correct, then all the $\mathrm{M}-\mathrm{H}$ curve should collapse onto two separate curves below and above $\mathrm{T}_{C}$ independently. The Eq. (12) can be rewritten in terms of the renormalized magnetization $\mathrm{m}\left[\mathrm{m}=\mathrm{M}\left(\mu_{0} \mathrm{H}, \epsilon\right) \epsilon^{-\beta}\right]$ and renormalized field $\mathrm{h}\left[\mathrm{h}=\mu_{0} \mathrm{H} \epsilon^{-(\beta+\gamma)}\right]$ as

$$
m=f_{ \pm}(h) \text {. }
$$

Furthermore, according to the statistical theory, the relations between the critical exponents that limit the number of independent variables to two are given as ${ }^{47,50,51,71}$ :

$$
\alpha+2 \beta+\gamma=2 \quad \text { (Rushbrooke scaling realtion) }
$$

and

$$
\left.\delta=1+\frac{\gamma}{\beta} . \quad \text { (Widom scaling realtion }\right)
$$

\section{Appendix B: Derivation of Eq. (1)}

The $\Delta S_{M}$ can be calculated by using Maxwell's thermodynamic relation given below ${ }^{39,43}$

$$
\begin{array}{r}
\Delta S_{M}\left(\mu_{0} H, T\right)=\Delta S_{M}\left(\mu_{0} H, T\right)-\Delta S_{M}(0, T), \\
\Delta S_{M}\left(\mu_{0} H, T\right)=\int_{0}^{\mu_{0} H_{\max }}\left(\frac{\partial S\left(\mu_{0} H, T\right)}{\partial\left(\mu_{0} H\right)}\right)_{T} d\left(\mu_{0} H\right) .
\end{array}
$$

Using Maxwell's thermodynamic relation

$$
\left(\frac{\partial S\left(\mu_{0} H, T\right)}{\partial\left(\mu_{0} H\right)}\right)_{T}=\left(\frac{\partial M\left(\mu_{0} H, T\right)}{\partial T}\right)_{\mu_{0} H} .
$$

Now, above relation $\Delta S_{M}$ can be expressed as follows

$$
\Delta S_{M}\left(\mu_{0} H, T\right)=\int_{0}^{\mu_{0} H_{\max }}\left(\frac{\partial M\left(\mu_{0} H, T\right)}{\partial T}\right)_{\mu_{0} H} d\left(\mu_{0} H\right) .
$$

Using isothermal M-H curves, the $\Delta S_{M}$ in the presence of the magnetic field can be calculated numerically from the following equation

$$
\begin{gathered}
\Delta S_{M}\left(\frac{T_{1}+T_{2}}{2}\right)=\frac{1}{\left(T_{1}-T_{2}\right)}\left[\int _ { 0 } ^ { \mu _ { 0 } H _ { \operatorname { m a x } } } \left(\partial M\left(\mu_{0} H, T_{2}\right)\right.\right. \\
\left.-\partial M\left(\mu_{0} H, T_{1}\right)_{\mu_{0} H} d\left(\mu_{0} H\right)\right] .
\end{gathered}
$$

Received: 10 December 2020; Accepted: 16 June 2021

Published online: 08 July 2021

\section{References}

1. Fawcett, I. D., Sunstrom, J. E. IV., Greenblatt, M., Croft, M. \& Ramanujachary, K. Structure, magnetism, and properties of Ruddlesden-Popper calcium manganates prepared from citrate gels. Chem. Mater. 10, 3643-3651 (1998).

2. Dagotto, E. Nanoscale phase separation and colossal magnetoresistance: the physics of manganites and related compounds, vol. 136 (Springer, 2013).

3. O’Reilly, T. J. \& Offenbacher, E. L. Molecular orbital calculation for trigonally distorted octahedral complexes. J. Chem. Phys. 54, 3065-3076 (1971).

4. Van Vleck, J. Theory of the variations in paramagnetic anisotropy among different salts of the iron group. Phys. Rev. 41, 208 (1932). 
5. Jahn, H. A. \& Teller, E. Stability of polyatomic molecules in degenerate electronic states-i-orbital degeneracy. Proc. R. Soc. Lond. 161, 220-235 (1937).

6. Zener, C. Interaction between the d shells in the transition metals. Phys. Rev. 81, 440 (1951).

7. Paraskevopoulos, M. et al. Magnetic properties and the phase diagram of la1-xsrxmno3 for x-0.2. J. Phys. Condens. Matter 12, 3993 (2000).

8. Hemberger, J. et al. Structural, magnetic, and electrical properties of single-crystalline la $1-\mathrm{x}$ sr $\mathrm{x}$ mno $3(0.4<\mathrm{x}<0.85)$. Phys. Rev. B 66, 094410 (2002).

9. Urushibara, A. et al. Insulator-metal transition and giant magnetoresistance in la 1-x sr x mno 3. Phys. Rev. B 51, 14103 (1995).

10. Rao, C. N. R. \& Raveau, B. Colossal magnetoresistance, charge ordering and related properties of manganese oxides. (World Scientific, 1998).

11. Szewczyk, A. et al. Magnetocaloric effect in la 1-x sr $\mathrm{x}$ mno 3 for $\mathrm{x}=0.13$ and 0.16. Appl. Phys. Lett. 77, 1026-1028 (2000).

12. Coey, J., Viret, M. \& Von Molnar, S. Mixed-valence manganites. Adv. Phys. 48, 167-293 (1999).

13. von Helmolt, R., Wecker, J., Holzapfel, B., Schultz, L. \& Samwer, K. Giant negative magnetoresistance in perovskitelike la $2 / 3$ ba 1/3 mno x ferromagnetic films. Phys. Rev. Lett. 71, 2331 (1993).

14. Jin, S. et al. Thousandfold change in resistivity in magnetoresistive La-Ca-Mn-O films. Science 264, 413-415 (1994).

15. Tokura, Y. et al. Giant magnetotransport phenomena in filling-controlled kondo lattice system: Lal-x sr x mno3. J. Phys. Soc. Jpn. 63, 3931-3935 (1994).

16. Chmaissem, O. et al. Structural and magnetic phase diagrams of la 1-x sr x mno 3 and pr 1-y sr y mno 3. Phys. Rev. B 67, 094431 (2003).

17. Kimura, T. et al. Interplane tunneling magnetoresistance in a layered manganite crystal. Science 274, 1698-1701 (1996).

18. Jonker, G. \& Van Santen, J. Ferromagnetic compounds of manganese with perovskite structure. Physica 16, 337-349 (1950).

19. Chahara, K.-I., Ohno, T., Kasai, M. \& Kozono, Y. Magnetoresistance in magnetic manganese oxide with intrinsic antiferromagnetic spin structure. Appl. Phys. Lett. 63, 1990-1992 (1993).

20. Rao, C. \& Cheetham, A. Charge ordering in manganates. Science 276, 911-913 (1997)

21. Wang, A., Liu, Y., Zhang, Z., Long, Y. \& Cao, G. Magnetic entropy change and colossal magnetoresistance effect in the layered perovskite la1. 34sr1. 66mn2o7. Solid State Commun. 130, 293-296 (2004).

22. Moritomo, Y., Asamitsu, A., Kuwahara, H. \& Tokura, Y. Giant magnetoresistance of manganese oxides with a layered perovskite structure. Nature 380, 141-144 (1996).

23. Asano, H., Hayakawa, J. \& Matsui, M. Two-dimensional ferromagnetic ordering and magnetoresistance in the layered perovskite la $2-2$ x ca $1+2$ x mn 2 o 7. Phys. Rev. B 56, 5395 (1997).

24. Seshadri, R. et al. Study of the layered magnetoresistive perovskite la1. $2 \mathrm{sr} 1.8 \mathrm{mn} 207$ by high-resolution electron microscopy and synchrotron x-ray powder diffraction. Chem. Mater. 9, 1778-1787 (1997).

25. Hirota, K. et al. Spin dynamical properties and orbital states of the layered perovskite la $2-2 \mathrm{x}$ sr $1+2 \mathrm{x} \mathrm{mn} 2$ o $7(0.3<\mathrm{x}<0.5)$. Phys. Rev. B 65, 064414 (2002).

26. Yu, X. et al. Biskyrmion states and their current-driven motion in a layered manganite. Nat. Commun. 5, 1-7 (2014).

27. Nagai, T. et al. Formation of nanoscale magnetic bubbles in ferromagnetic insulating manganite la $7 / 8 \mathrm{sr} 1 / 8 \mathrm{mno}$. Appl. Phys. Lett. 101, 162401 (2012).

28. Yu, X., Tokunaga, Y., Taguchi, Y. \& Tokura, Y. Variation of topology in magnetic bubbles in a colossal magnetoresistive manganite. Adv. Mater. 29, 1603958 (2017).

29. Morikawa, D. et al. Lorentz transmission electron microscopy on nanometric magnetic bubbles and skyrmions in bilayered manganites la1. 2sr1. 8 (mn1- yruy) 207 with controlled magnetic anisotropy. Appl. Phys. Lett. 107, 212401 (2015).

30. Nagaosa, N. \& Tokura, Y. Topological properties and dynamics of magnetic skyrmions. Nat. Nanotechnol. 8, 899-911 (2013).

31. Fert, A., Cros, V. \& Sampaio, J. Skyrmions on the track. Nat. Nanotechnol. 8, 152-156 (2013).

32. Chauhan, H. C., Kumar, B., Tiwari, J. K. \& Ghosh, S. Multiple phases with a tricritical point and a lifshitz point in the skyrmion host $\mathrm{Cu}_{2} \mathrm{OSeO}_{3}$. Phys. Rev. B 100, 165143 (2019).

33. Yu, X. et al. Near room-temperature formation of a skyrmion crystal in thin-films of the helimagnet FeGe. Nat. Mater. 10, 106-109 (2011).

34. Mahesh, R., Mahendiran, R., Raychaudhuri, A. \& Rao, C. Effect of dimensionality on the giant magnetoresistance of the manganates: a study of the (la, sr) $)_{n+1} \mathrm{Mn}_{n} \mathrm{O}_{n+1}$ family. J. Solid State Chem. 122, 448-450 (1996).

35. Jung, W.-H. Electrical transport properties of la2. 1sr1. 9mn3o10 system. J. Mater. Sci. Lett. 18, 967-970 (1999).

36. Wang, A. et al. Magnetic entropy change of the layered perovskites la 2-2 x sr 1+ 2 x mn 2 o 7. J. Appl. Phys. 97, 103906 (2005).

37. Argyriou, D. et al. Unconventional magnetostriction in layered $\mathrm{La}_{1} \cdot 2 \mathrm{Sr}_{1} .8 \mathrm{Mn}_{2} \mathrm{O}_{7}$ : evidence for spin-lattice coupling above $\mathrm{T}_{C}$. Phys. Rev. B 55, R11965 (1997).

38. Franco, V., Conde, A., Romero-Enrique, J. \& Blázquez, J. A universal curve for the magnetocaloric effect: an analysis based on scaling relations. J. Phys. Condens. Matter 20, 285207 (2008).

39. Romero-Muniz, C., Tamura, R., Tanaka, S. \& Franco, V. Applicability of scaling behavior and power laws in the analysis of the magnetocaloric effect in second-order phase transition materials. Phys. Rev. B 94, 134401 (2016).

40. Franco, V., Conde, A., Pecharsky, V. \& Gschneidner, K. Jr. Field dependence of the magnetocaloric effect in Gd and (Er1- xDyx) al2: does a universal curve exist?. Europhys. Lett. 79, 47009 (2007).

41. Bonilla, C. M. et al. Universal behavior for magnetic entropy change in magnetocaloric materials: an analysis on the nature of phase transitions. Phys. Rev. B 81, 224424 (2010).

42. Guetari, R., Bartoli, T., Cizmas, C., Mliki, N. \& Bessais, L. Structure, magnetic and magnetocaloric properties of new nanocrystalline (pr, dy) Fe9 compounds. J. Alloys Compd. 684, 291-298 (2016).

43. Bingham, N., Phan, M., Srikanth, H., Torija, M. \& Leighton, C. Magnetocaloric effect and refrigerant capacity in charge-ordered manganites. J. Appl. Phys. 106, 023909 (2009).

44. Domb, C. Phase transitions and critical phenomena (Elsevier, 2000).

45. Banerjee, B. On a generalised approach to first and second order magnetic transitions. Phys. Lett. 12, 16-17 (1964).

46. Arrott, A. \& Noakes, J. E. Approximate equation of state for nickel near its critical temperature. Phys. Rev. Lett. 19, 786 (1967).

47. Widom, B. Surface tension and molecular correlations near the critical point. J. Chem. Phys. 43, 3892-3897 (1965).

48. Kouvel, J. S. \& Fisher, M. E. Detailed magnetic behavior of nickel near its curie point. Phys. Rev. 136, A1626 (1964).

49. Kim, D., Zink, B., Hellman, F. \& Coey, J. Critical behavior of $\mathrm{La}_{0} .75 \mathrm{Sr}_{0} .25 \mathrm{Mno}_{3}$. Phys. Rev. B 65, 214424 (2002).

50. Kadanoff, L. P. Scaling laws for ising models near $T_{C}$. Phys. Phys. Fiz. 2, 263 (1966).

51. Huang, K. Statistical mechanics, 2nd edn. (1987).

52. Fisher, M. E. The renormalization group in the theory of critical behavior. Rev. Mod. Phys. 46, 597 (1974).

53. Fischer, S., Kaul, S. \& Kronmüller, H. Critical magnetic properties of disordered polycrystalline $\mathrm{Cr}_{7} 5 \mathrm{Fe}_{2} 5$ and $\mathrm{Cr}_{7} 0 \mathrm{Fe}_{3} \mathrm{O}$ alloys. Phys. Rev. B 65, 064443 (2002).

54. Taroni, A., Bramwell, S. T. \& Holdsworth, P. C. Universal window for two-dimensional critical exponents. J. Phys. Condens. Matter 20, 275233 (2008).

55. Osborn, R. et al. Neutron scattering investigation of magnetic bilayer correlations in $\mathrm{La}_{1} .2 \mathrm{Sr}_{1} .8 \mathrm{Mn}_{2} \mathrm{O}_{7}$ : evidence of canting above TC. Phys. Rev. Lett. 81, 3964 (1998).

56. Gordon, J., Bader, S., Mitchell, J., Osborn, R. \& Rosenkranz, S. Specific heat of $\mathrm{La}_{1} .2 \mathrm{Sr}_{1} .8 \mathrm{Mn}_{2} \mathrm{O}_{7}$. Phys. Rev. B 60, 6258 (1999). 
57. Shi, J., Wu, F. \& Lin, C. Electrical transport, magnetism, and magnetoresistance in $\mathrm{La}_{0} .7 \mathrm{Sr}_{0} .3(\mathrm{Mn1}-\mathrm{xcox}) \mathrm{O}_{3}$. Appl. Phys. A 68, 577-581 (1999).

58. Heffner, R. et al. Effects of reduced dimensionality on spin dynamics in the layered perovskite $\mathrm{La}_{1} \cdot 4 \mathrm{Sr}_{1} \cdot 6 \mathrm{Mn}_{2} \mathrm{O}_{7}$. Phys. Rev. Lett. 81, 1706 (1998).

59. Sonomura, H., Terai, T., Kakeshita, T., Osakabe, T. \& Kakurai, K. Neutron diffraction study on magnetic structures in a La 1.37 $\mathrm{Sr}_{1} .63 \mathrm{Mn}_{2} \mathrm{O}_{7}$ single crystal under hydrostatic pressures of up to $0.8 \mathrm{Gpa}$. Phys. Rev. B 87, 184419 (2013).

60. Fujioka, H. et al. Spin dynamical properties of the layered perovskite $\mathrm{La}_{1} .2 \mathrm{Sr}_{1} .8 \mathrm{Mn}_{2} \mathrm{O}_{7}$. J. Phys. Chem. Solids 60, 1165-1168 (1999).

61. Martin, M. C. et al. Magnetism and structural distortion in the $\mathrm{La}_{0} \cdot 7 \mathrm{Sr}_{0} \cdot 3 \mathrm{MnO}_{3}$ metallic ferromagnet. Phys. Rev. B 53, 14285 (1996).

62. Vasiliu-Doloc, L., Lynn, J., Mukovskii, Y., Arsenov, A. \& Shulyatev, D. Spin dynamics of strongly doped Spin dynamics of strongly doped $\mathrm{La}_{1-x} \mathrm{Sr}_{x} \mathrm{MnO}_{3}$. J. Appl. Phys. 83, 7342-7344 (1998).

63. Halperin, B. I. \& Hohenberg, P. Generalization of scaling laws to dynamical properties of a system near its critical point. Phys. Rev. Lett. 19, 700 (1967).

64. Halperin, B. \& Hohenberg, P. Scaling laws for dynamic critical phenomena. Phys. Rev. 177, 952 (1969).

65. Halperin, B., Hohenberg, P. \& Ma, S.-K. Calculation of dynamic critical properties using Wilson's expansion methods. Phys. Rev. Lett. 29, 1548 (1972).

66. Halperin, B., Hohenberg, P. \& Ma, S.-K. Renormalization-group methods for critical dynamics: I. Recursion relations and effects of energy conservation. Phys. Rev. B 10, 139 (1974).

67. Halperin, B., Hohenberg, P. \& Ma, S.-K. Renormalization-group methods for critical dynamics: II. Detailed analysis of the relaxational models. Phys. Rev. B 13, 4119 (1976).

68. Hohenberg, P. C. \& Halperin, B. I. Theory of dynamic critical phenomena. Rev. Mod. Phys. 49, 435 (1977).

69. Mazenko, G. F. Nonequilibrium statistical mechanics (Wiley, London, 2008).

70. Ginzburg, V. Some remarks on phase transitions of the second kind and the microscopic theory of ferroelectric materials. Soviet Phys. Solid State 2, 1824-1834 (1961).

71. Stanley, H. Introduction to phase transitions and critical phenomena. (Oxford University Press, Oxford, 1971).

72. Fisher, M. E. The theory of equilibrium critical phenomena. Rep. Prog. Phys. 30, 615 (1967).

73. Stanley, H. E. Scaling, universality, and renormalization: three pillars of modern critical phenomena. Rev. Mod. Phys. 71, S358 (1999).

74. Kaul, S. Static critical phenomena in ferromagnets with quenched disorder. J. Magn. Magn. Mater. 53, 5-53 (1985).

\section{Acknowledgements}

We thank AIRF-JNU for providing facilities for PPMS and XRD measurement. We also thank the "Low temperature and high magnetic field facility" at the Central University of Rajasthan. J.K.T. acknowledges UGC, India for financial support through fellowship. This project is partially supported by DST-PURSE Government of India.

\section{Author contributions}

The first author (J.K.T.) has done the data analysis and wrote the main manuscript text. All the authors have discussed the data analysis and reviewed the manuscript.

\section{Competing interests}

The authors declare no competing interests.

\section{Additional information}

Correspondence and requests for materials should be addressed to S.G.

Reprints and permissions information is available at www.nature.com/reprints.

Publisher's note Springer Nature remains neutral with regard to jurisdictional claims in published maps and institutional affiliations.

(c) (i) Open Access This article is licensed under a Creative Commons Attribution 4.0 International

License, which permits use, sharing, adaptation, distribution and reproduction in any medium or format, as long as you give appropriate credit to the original author(s) and the source, provide a link to the Creative Commons licence, and indicate if changes were made. The images or other third party material in this article are included in the article's Creative Commons licence, unless indicated otherwise in a credit line to the material. If material is not included in the article's Creative Commons licence and your intended use is not permitted by statutory regulation or exceeds the permitted use, you will need to obtain permission directly from the copyright holder. To view a copy of this licence, visit http://creativecommons.org/licenses/by/4.0/.

(c) The Author(s) 2021 